\title{
Does preimplantation genetic testing for aneuploidy really improve IVF outcomes in advanced maternal age patients without compromising cumulative live-birth rate?
}

\author{
Raoul Orvieto ${ }^{1,2,3}$ \\ Received: 19 November 2019 / Accepted: 5 December 2019/Published online: 11 December 2019 \\ (C) Springer Science+Business Media, LLC, part of Springer Nature 2019
}

Dear Editor,

We read with interest the study by Sacchi et al. [1], reporting on a single-center observational-cohort study with 2 years' follow-up, of couples with female patients between 38 and 44 years of age undergoing PGT-A. Compared with control group, patients undergoing PGT-A showed improved clinical outcomes with no negative impact of PGT-A-related interventions on cumulative delivery rate $(26.3 \%, 95 \% \mathrm{CI}$ $21.5-31.6$ vs $24.0 \%, 95 \%$ CI $22.5-25.6$ for PGT-A and control, respectively).

While their findings are interesting, the results as presented are quite misleading. A closer look at their results revealed that only patients with FSH levels $<12 \mathrm{mIU} / \mathrm{mL}$ and/or AMH levels $>0.5 \mathrm{ng} / \mathrm{mL}$, yielding $>10$ oocytes were included, excluding poor ovarian response (POR). Moreover, 370 couples were enrolled to the PGT-A group vs 2168 to the control group - these are the figures that should appear in the denominators when calculating ongoing pregnancy rate (OPR). Recalculation of the cumulative OPR reveals a significant difference in favor of the control group [902/2168 (41.6\%) vs 84/ $370(22.7 \%), p<0.0000]$. Figures are in agreement with the previously published hypothetical RCT [2].

Furthermore, taking into consideration that the prevalence of POR is above $10 \%$ in the general IVF population and increases with age to approximately $50 \%$ in women over 40 years of age [3], offering PGT-A to advanced age patients

Raoul Orvieto

raoul.orvieto@sheba.health.gov.il

1 Infertility and IVF Unit, Department of Obstetrics and Gynecology, Chaim Sheba Medical Center (Tel Hashomer), Ramat Gan, Israel

2 Sackler Faculty of Medicine, Tel Aviv University, Tel Aviv, Israel

3 The Tarnesby-Tarnowski Chair for Family Planning and Fertility Regulation, Sackler Faculty of Medicine, Tel-Aviv University, Tel Aviv, Israel (including POR) would be even more deleterious. Based on Sacchi et al. [1] study, the 370 couples enrolled were probably chosen from a larger cohort of patients, probably 500 couples, if taking $25 \%$ as an average prevalence of POR in this age group. Therefore, cumulative OPR would have been even lower [84/500 (16.8\%)], figures that should avoid offering PGT-A to this age group [4].

To conclude, this study again confirms the facts that in unfavorable patient populations (advanced age or POR), who were a priori considered as the best candidates for PGTA, offering PGT-A may actually reduce pregnancy and live birth chances, and should not be offered in association with IVF.

\section{References}

1. Sacchi L, Albani E, Cesana A, Smeraldi A, Parini V, Fabiani M, et al. Preimplantation genetic testing for aneuploidy improves clinical, gestational, and neonatal outcomes in advanced maternal age patients without compromising cumulative live-birth rate. J Assist Reprod Genet. 2019:1-12. https://doi.org/10.1007/s10815-01901609-4.

2. Orvieto R. Preimplantation genetic screening - the required RCT that has not yet been carried out. Reprod Biol Endocrinol. 2016;14:35.

3. Ferraretti AP, La Marca A, Fauser BC, Tarlatzis B, Nargund G, Gianaroli L, et al. ESHRE consensus on the definition of "poor response' to ovarian stimulation for in vitro fertilization: the Bologna criteria. Hum Reprod. 2011;26:1616-24.

4. Gleicher N, Orvieto R. Is the hypothesis of preimplantation genetic screening (PGS) still supportable? A review. J Ovarian Res. 2017;10(1):21.

Publisher's note Springer Nature remains neutral with regard to jurisdictional claims in published maps and institutional affiliations. 